\title{
Trunk Pitch Oscillations for Joint Load Redistribution in Humans and Humanoid Robots
}

\author{
Özge Drama and Alexander Badri-Spröwitz
}

\begin{abstract}
Creating natural-looking running gaits for humanoid robots is a complex task due to the underactuated degree of freedom in the trunk, which makes the motion planning and control difficult. The research on trunk movements in human locomotion is insufficient, and no formalism is known to transfer human motion patterns onto robots. Related work mostly focuses on the lower extremities, and simplifies the problem by stabilizing the trunk at a fixed angle. In contrast, humans display significant trunk motions that follow the natural dynamics of the gait. In this work, we use a springloaded inverted pendulum model with a trunk (TSLIP) together with a virtual point (VP) target to create trunk oscillations and investigate the impact of these movements. We analyze how the VP location and forward speed determine the direction and magnitude of the trunk oscillations. We show that positioning the VP below the center of mass $(\mathrm{CoM})$ can explain the forward trunk pitching observed in human running. The VP below the CoM leads to a synergistic work between the hip and leg, reducing the leg loading. However, it comes at the cost of increased peak hip torque. Our results provide insights for leveraging the trunk motion to redistribute joint loads and potentially improve the energy efficiency in humanoid robots.
\end{abstract}

\section{INTRODUCTION}

Traditional humanoid research considers trunk movements as undesired and designs controllers to keep the trunk at a fixed angle [1,2], which leads to stiff and fixed upper bodies. Fixed trunk postures are in sharp contrast with the human locomotion, that exhibits pronounced trunk oscillations of up to $\pm 2-6$ in the sagittal plane [3-7]. The trunk matters in bipedal anatomy, as it is inherently unstable, comprises $50 \%$ of total body mass, and has a large inertia $[7,8]$. Thus, even small deviations in trunk position can significantly effect the whole body dynamics [3]. In this paper, we use a springloaded inverted pendulum model with a controller based on a virtual point (VP) to generate trunk pitch oscillations with varying magnitudes and direction. We investigate how the trunk angular excursion and the direction of the oscillation depend on the VP position and the running speed.

Humans utilize the trunk's inertia extensively to assist locomotion; especially during running to offset the highly accelerated swing motion and counteract destabilizing, high ground reaction forces (GRF) [9]. In running, the human trunk pitches forward (anteriorly) during the first half of the stance phase, reaches a maximum flexion at mid-stance and pitches backward (posteriorly) in the second half of the stance phase $[4,5,7]$. In walking, the trunk moves forward

The authors thank the International Max Planck Research School for Intelligent Systems (IMPRS-IS) for supporting Özge Drama.

Özge Drama and Alexander Badri-Spröwitz are with the Dynamic Locomotion Group at Max Planck Institute for Intelligent Systems, Stuttgart, Germany $\{$ drama, sprowitz\}@is.mpg.de in the double stance phase. These oscillations can be characterized by four parameters (i)-(iv) [4], which are adjusted in response to variations in speed, mode of progression (e.g., gait type, motion direction) and terrain conditions.

(i) Mean trunk inclination

(ii) Trunk angular excursion (i.e., net angular displacement)

(iii) Mean trunk angular velocity

(iv) The phase of max. trunk flexion w.r.t. the stride cycle

Experiments on human running show that for speeds of $2.5-6 \mathrm{~m} \mathrm{~s}^{-1}$, the mean trunk inclination increases from 8 to $14^{\circ}[6,10]$, along with a trunk angular excursion that extends from 2.5 to $5^{\circ}[6,7]$ and a mean trunk angular velocity that increases from 5 to $35^{\circ} \mathrm{s}^{-1}$ [4]. Meanwhile, the phase of maximum trunk flexion shifts from 30 to $20 \%$ of the strike cycle; so that the maximum trunk lean occurs closer to the aerial phase as the speed increases [4]. These observations indicate that humans might utilize the trunks inertia and its oscillations to achieve their impressive locomotor energetics, speed, and robustness. Humanoid robots could similarly benefit from human-inspired trunk pitch strategies [11].

The spring-loaded inverted pendulum (SLIP) model captures the essential characteristics of running. It consists of a point-mass body attached to a massless, springy leg [12]. This model can be extended with a rigid trunk (TSLIP), which is actuated by a torque at the hip $[13,14]$. A recent method to determine the hip torque is based on the VP concept, where the ground reaction forces are redirected to intersect at a point above the center of mass (CoM). This phenomenon has been observed experimentally in human walking [14-16], running [13], and other animal gaits $[17,18]$. Previous research often interprets the VP as a pivot point that provides a pendulum-like support and implements it as the target variable of control to achieve postural stability. In contrast to a $\mathrm{VP}$ above the $\mathrm{CoM}\left(\mathrm{VP}_{\mathrm{A}}\right)$, a recent study with parkinson patients provides evidence to a VP below $\mathrm{CoM}\left(\mathrm{VP}_{\mathrm{B}}\right)$ in walking gaits [19]. As an important novelty, here we focus our research on the effect of VP location on trunk oscillations.

Most humanoid robots stabilize their trunk position at a fixed angle without any trunk movements $[1,2]$. An exception are the robots WL-12RV and Honda ASIMO, which create trunk accelerations to address unstable dynamic situations [20,21]. The control strategy in ASIMO extends static walking and uses the trunk reaction to recover stability while running. Another approach is shown in the ATRIAS robot, which implements a VP target [22] or a sinusoidal reference trajectory [23] to create trunk movements for walking. While 
this approach works in practice, it is not well understood how the reference for the VP affect the whole body motion.

In this work, we implement a TSLIP model with different VP targets. We then systematically compare the resulting gaits for the speeds of $4-10 \mathrm{~ms}^{-1}$, which is the physical limit for human running [9]. We show that

(a) VP above the CoM $\left(\mathrm{VP}_{\mathrm{A}}\right)$ generates backward trunk motion that is observed in the single stance phase of human walking $[3,7]$; whereas a VP below the CoM $\left(\mathrm{VP}_{\mathrm{B}}\right)$ causes forward motion that is seen in running [7];

(b) $\mathrm{VP}_{\mathrm{A}}$ results in smaller trunk angular excursions, as opposed to $\mathrm{VP}_{\mathrm{B}}$ with the same $\mathrm{VP}$ radius (i.e., the same distance between VP and CoM);

(c) the trunk angular excursion and mean trunk angular velocity increase with speed for the same VP radius.

We demonstrate that the standard TSLIP model is not capable of predicting leg length velocity accurately, and propose a bilinear damper to obtain smooth force profiles. Finally, our findings support the hypothesis that trunk motions can be used in favor of energy economy. We observe that a $\mathrm{VP}_{\mathrm{B}}$ reduces the leg loading, which makes it appealing to place the VP further down. However, a lower VP shifts the power requirements to the hip, and increases peak hip torques. Therefore there is a trade-off between leg loading and the peak torque when choosing the VP location.

\section{SIMULATION MODEL}

In this section, we describe our TSLIP model applied in this work. The TSLIP model consists of a trunk with mass $m$ and moment of inertia $J$, which are attached to a massless leg at the hip. The leg is equipped with a parallel springdamper mechanism (Fig. 1). The system's hybrid dynamics are characterized by a flight phase, where the CoM moves in a ballistic motion; and a stance phase, where the leg force and hip torque propel the body. The transition between these phases occurs at touch-down (TD) when the foot point comes in contact with the ground, and take-off (TO) when the GRF becomes zero or the leg reaches its rest length $l_{0}$.

The equations of the motion for the CoM state $\left(x_{C}, y_{C}, \theta_{C}\right)$

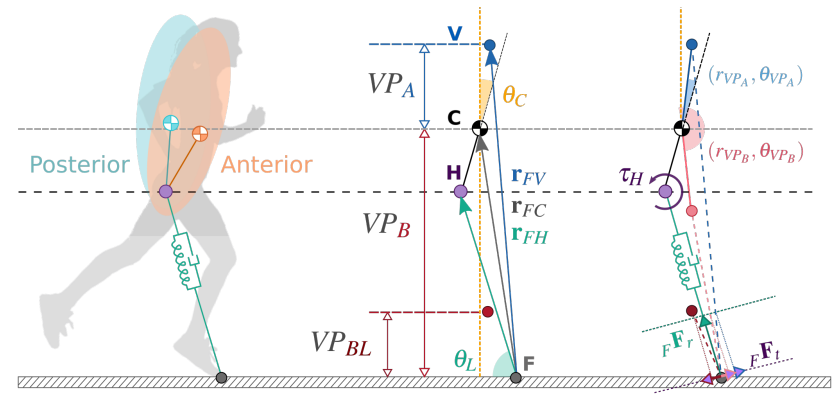

Fig. 1. TSLIP model and vector definitions for the human morphology. region demonstrates clockwise trunk rotation, whereas is the opposite rotation. The letters $\mathrm{V}, \mathrm{C}, \mathrm{H}$ denote the virtual point, $\mathrm{CoM}$, hip respectively. The position vectors between points are referred as $\mathbf{r}_{F H}, \mathbf{r}_{F V}, \mathbf{r}_{F C}, \mathbf{r}_{F H}$. The angles $\theta_{L}, \theta_{C}, \theta_{V P}$ are the leg, trunk and VP angles. during the stance phase can be written as,

$$
m\left[\begin{array}{c}
\ddot{x}_{C} \\
\ddot{y}_{C}
\end{array}\right]={ }_{F} \mathbf{F}_{a}+{ }_{F} \mathbf{F}_{t}+g \text { and } J \ddot{\theta}_{C}=-\mathbf{r}_{F C} \times\left({ }_{F} \mathbf{F}_{a}+{ }_{F} \mathbf{F}_{t}\right),
$$

where the leg spring-damper forces $F_{s p}$ and $F_{d p}$ generate the axial component of the GRF in foot frame ${ }_{F} \mathbf{F}_{a}$, and the hip torque $\tau_{H}$ generates the tangential component ${ }_{F} \mathbf{F}_{t}$,

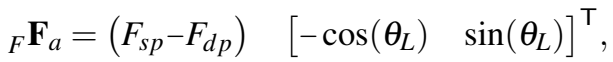

$$
\begin{aligned}
& { }_{F} \mathbf{F}_{t}=\left(\begin{array}{lll}
\left.-\tau_{H} / l_{L}\right) & {\left[\sin \left(\theta_{L}\right)\right.} & -\cos \left(\theta_{L}\right)
\end{array}\right]^{\top} .
\end{aligned}
$$

We implemented a linear leg spring, with force $F_{s p}=k\left(l_{0}-l\right)$, with spring constant $k$, leg length $l$, and leg rest length $l_{0}$. The damping is often chosen to be linear; we explain our choice of nonlinear damping $\left(F_{d p}\right)$ in Sec. III

Since the leg is passively compliant, trunk pitch motions can only be controlled via the hip torque $\tau_{H}$. We select $\tau_{H}$, such that the GRF points to a VP, which is characterized by a radius $r_{V P}$, and an angle $\theta_{V P}$ relative to the CoM (Fig. 1),

$$
\begin{gathered}
\tau_{H}=\tau_{V P}={ }_{F} \mathbf{F}_{a} \times\left[\frac{\mathbf{r}_{F V} \times \mathbf{r}_{F H}}{\mathbf{r}_{F V} \cdot \mathbf{r}_{F H}}\right] \times l, \\
\mathbf{r}_{F V}=\mathbf{r}_{F C}+r_{V P}\left[\begin{array}{r}
-\sin \left(\theta_{C}+\theta_{V P}\right) \\
\cos \left(\theta_{C}+\theta_{V P}\right)
\end{array}\right] .
\end{gathered}
$$

Our model has seven morphological parameters, which are selected to match a human of $80 \mathrm{~kg}$, with $1 \mathrm{~m}$ leg (Table \).

TABLE I. Model parameters for TSLIP model

\begin{tabular}{l|ccccc} 
Name & Symbol & Units & Literature & Chosen & Reference \\
\hline mass & $m$ & $\mathrm{~kg}$ & $60-80$ & 80 & {$[14,24]$} \\
moment of inertia & $J$ & $\mathrm{~kg} \mathrm{~m}^{2}$ & 5 & 5 & {$[8,24]$} \\
leg stiffness & $k$ & $\mathrm{kNm}^{-1}$ & $16-26$ & 18 & {$[24,25]$} \\
leg length & $l_{0}$ & $\mathrm{~m}$ & 1 & 1 & {$[14,24]$} \\
leg angle at TD & $\theta_{L}^{T D}$ & $\left(^{\circ}\right)$ & $78-71$ & $f_{H}(\dot{x})$ & {$[24,25]$} \\
dist. Hip-CoM & $r_{H C}$ & $\mathrm{~m}$ & 0.1 & 0.1 & {$[24,26]$}
\end{tabular}

\section{MODIFICATIONS}

In this section, we modify the leg damping to match the human data and introduce a novel control scheme that creates a range of feasible running gaits and trunk oscillations.

1) Model Modifications: In a TSLIP simulation with no other energy sinks, linear leg dampers are often introduced to absorb the energy inserted by the hip torque [17,24]. However, this leads to non-zero leg length velocities at $\mathrm{TD} / \mathrm{TO}$, and thus discontinuous leg forces ( $\boldsymbol{\Lambda}$, Fig. 2). In contrast, this is not observed in humans (o). To obtain realistic behavior, we use a bilinear damping with force

$$
F_{d p}=c i\left(l_{0}-l\right)=c i \Delta l
$$

where $i$ is the leg length velocity and $c$ is the damping coefficient. This damping leads to smooth damping forces ( $(\square)$ and is mathematically similar to the hill-type muscle model [27]. 


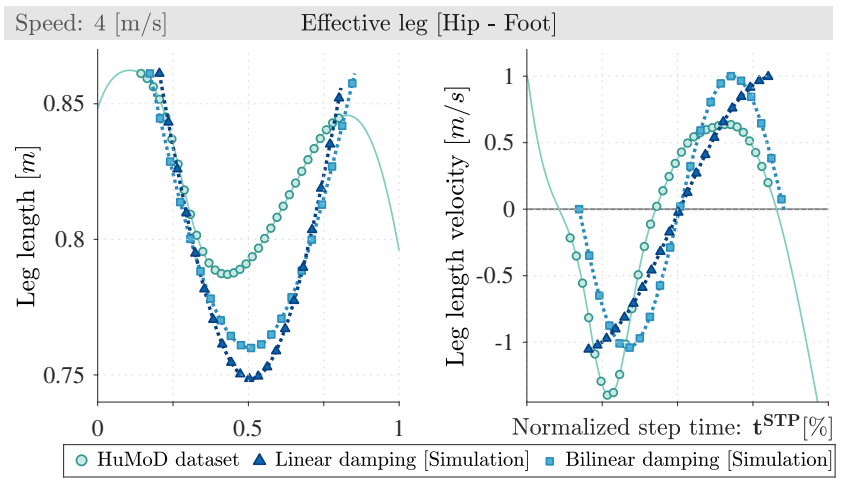

Fig. 2. A linear leg damper causes non-zero leg length velocities at TD/TO, which yield discontinuous damping forces. We introduce a bilinear damper to obtain more human-like behavior. The leg lengths are offset to the same TD value and velocities are normalized. The biomechanical data is estimated from the HuMoD dataset [26] using de Leva [8] body segment parameters.

2) Control Strategy: To regulate the leg angle at $\mathrm{TD}, \theta_{L}^{T D}$, we extend the method developed by [1]. In particular, at the $i$ th step, we choose

$\left.\theta_{L}^{T D}\right|_{i}=\left.\theta_{L}^{T D}\right|_{i-1}+k_{\dot{x} 0}\left(\left.\Delta \dot{x}_{C}^{A P}\right|_{0} ^{i}\right)+k_{\dot{x}}\left(\left.\Delta \dot{x}_{C}^{A P}\right|_{i-1} ^{i}\right)+k_{y}\left(\left.\Delta y_{C}^{A P}\right|_{i-1} ^{i}\right)$,

where $k_{\dot{x} 0}, k_{\dot{x}}$, and $k_{y}$ are the controller gains. Here, $\left.\Delta \dot{x}\right|_{0} ^{i}$ is the difference in apex velocity $\dot{x}$ between time steps 0 and $i$.

To generate trunk oscillations, we set the hip torque using a VP as in Eq. (3). However, this method is not robust and is highly sensitive to initial conditions and parameter variations [24]. One way to overcome this is to modify $\left(r_{V P}, \theta_{V P}\right)$ at every time step using, for example, a linear quadratic regulator control [24]. However, this controller can converge to any arbitrary $r_{V P}$ and is not suitable for our purpose.

In this work, we want to investigate the impact of a fixed $r_{V P}$ on the magnitude and direction of trunk oscillations. To this end, we propose a new incremental control strategy that facilitates VP gaits for a fixed $r_{V P}$ starting from various initial condition and for any model parameters.

First, we stabilize the VP controller with an additive PID term, $\tau_{H}=\tau_{V P}+\tau_{P I D}$, where

$$
\tau_{P I D}=k_{p}\left(\theta_{C}^{\text {Des }}-\theta_{C}\right)+k_{d}\left(\dot{\theta}_{C}^{\text {Des }}-\dot{\theta}_{C}\right)+k_{i}\left(\theta_{C}^{E r r}\right)
$$

with desired mean body pitch $\theta_{C}^{\text {Des }}$, pitch rate $\dot{\theta}_{C}^{\text {Des }}$, pitch integral error $\theta_{C}^{E r r}$, and control gains $k_{p}, k_{d}, k_{i}$. Applying this controller to the TSLIP model for two opposing positions of the VP (above-below) leads to a diverted GRF (Fig. 3, center column). The dotted lines represent the GRF for clockwise ( $\odot$ ) and counterclockwise (๑) trunk rotation. The magnitude of the GRF is plotted with red/blue lines at the bottom. The VP+PID controller does not focus the GRF at a single point as desired, but instead keeps the GRF close to a fixed point.

After the VP+PID controller converges to steady-state motion, we impose the GRF focus on a fixed point by disabling the PID, i.e., $\tau_{P I D}=0$, and adapting the VP angle,

$$
\left.\theta_{V P}\right|_{i}=\left.\theta_{V P}\right|_{i-1}+k_{v p}\left(\theta_{C}^{\text {Des }}-\Delta \theta_{C}\right) .
$$

This controller adjusts the VP angle based on the difference between the desired mean body angle $\theta_{C}^{\text {Des }}$ and the

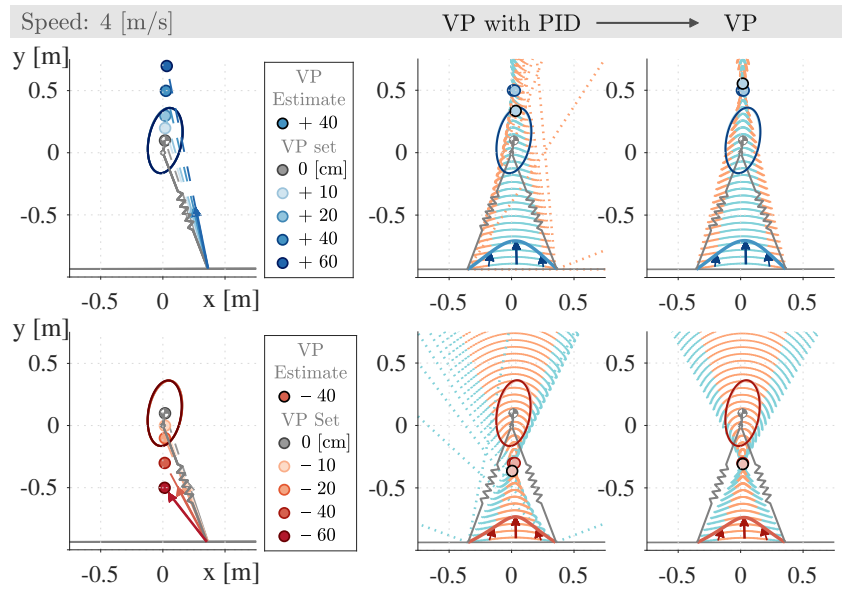

Fig. 3. Experimental setup (on left) and resulting GRF patterns with Eq Eq. 5) and without Eq. (6) PID reinforcement are plotted in non-rotating hip frame. The estimated VP is marked with black rimmed circles.

observed one over the last step, $\Delta \theta_{C}$. It converges to a fixed VP solution, where we can compare the pitch oscillation characteristics (Fig. 3, right column).

\section{SIMULATION RESULTS}

In this section, we describe our simulation setup to compare trunk oscillations and analyze our results.

\section{A. Simulation Setup}

In our simulations, we want to evaluate trunk oscillations up to at least $5^{\circ}$, as observed in humans. For this reason, we sweep VP targets over $r_{V P}= \pm[0,20,40,60] \mathrm{cm}$ and keep the desired mean body pitch angle fixed to $10^{\circ}$ (Fig. 3 . left column). We define the target VP angle w.r.t. body coordinates for $\mathrm{VP}_{\mathrm{A}}$ and w.r.t. world coordinates for $\mathrm{VP}_{\mathrm{B}}$.

Besides the VP location, the choice of control and damping parameters influence the flow of the motion. In biomechanics, gaits can be characterized by the duty factor (DF). $\mathrm{DF}$ is the ratio of the leg contact time to the stride period and is correlated to the magnitude of the GRF [28]. We tune control gains to match DFs of simulated gaits to the data in

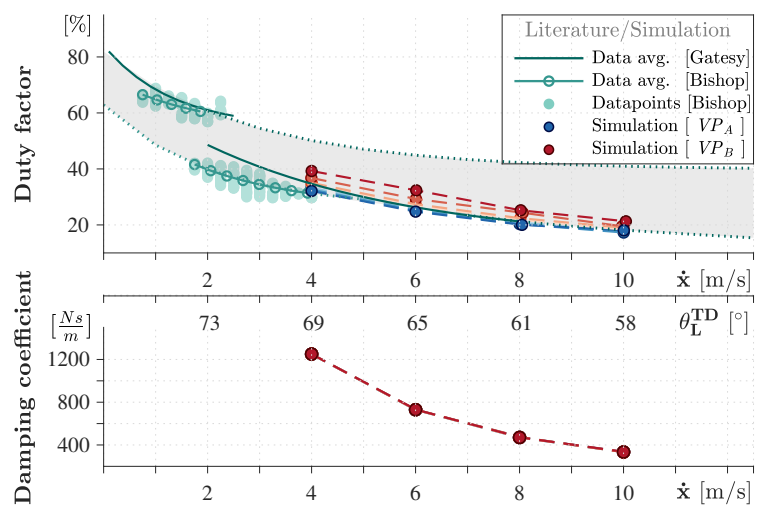

Fig. 4. Control parameters and damping are adjusted so that the simulation's duty factor lie in the grey shaded region estimated from biomechanical experiments $[28,29]$. 
$[28,29]$. Consequently, the DF, leg angle at TD, and damping coefficient decrease with speed (Fig. 4). We apply the same damping coefficient for all VP targets of equal speed.

\section{B. Analysis of the Ground Reaction Force (GRF)}

1) Effect of Damping: We investigate how the damping in our model affects the system dynamics. We subtract the component of GRF produced by damping (Fig. 5, lightcolored lines) and detect a phase shift to left when damping is present (Fig. 5 dark-colored lines, horizontal arrows).

The area under the horizontal GRF $\left(\mathrm{GRF}_{\mathrm{X}}\right)$ represents the fore-aft impulse, which is proportional to the forward acc/deceleration. Damping contributes to braking during the TD-MS phase (MS is the midstance) and hinders accelerating over the MS-TO phase. The damping contribution can be seen with the decrement in $\mathrm{GRF}_{\mathrm{x}}$ magnitude, which is denoted with vertical arrows (Fig. 5, right column).

2) Effect of VP Location: We compare three VP locations: VP at the CoM, above and below it. The VP location affects the decomposition of the GRF vector, and implicitly determines the cooperation between the leg force and force generated by the hip torque. When $\mathrm{VP}_{\mathrm{B}}$, leg and hip forces contribute to the $\mathrm{GRF}_{\mathrm{x}}$ synergistically and $\mathrm{GRF}_{\mathrm{y}}$ antagonistically, which is illustrated with blue/green arrows (Fig. 1 . right column). The $\mathrm{GRF}_{\mathrm{y}}$ is created mainly by the leg force, and the contribution of hip is negligible (Fig. 5, bottom left). On the other hand, the forces constructing $G F_{x}$ have a similar order of magnitude. $\mathrm{VP}_{\mathrm{B}}$ makes the hip force act along the same direction as the leg, and hence decreases the magnitude of $\mathrm{GRF}_{\mathrm{x}}$, as indicated with the red vertical arrows Fig. 5, bottom right). In other words, $\mathrm{VP}_{\mathrm{B}}$ causes the system to accelerate and decelerate more in the fore-aft direction. The $\mathrm{VP}_{\mathrm{A}}$ has the inverse effect and causes smaller accelerations/decelerations.

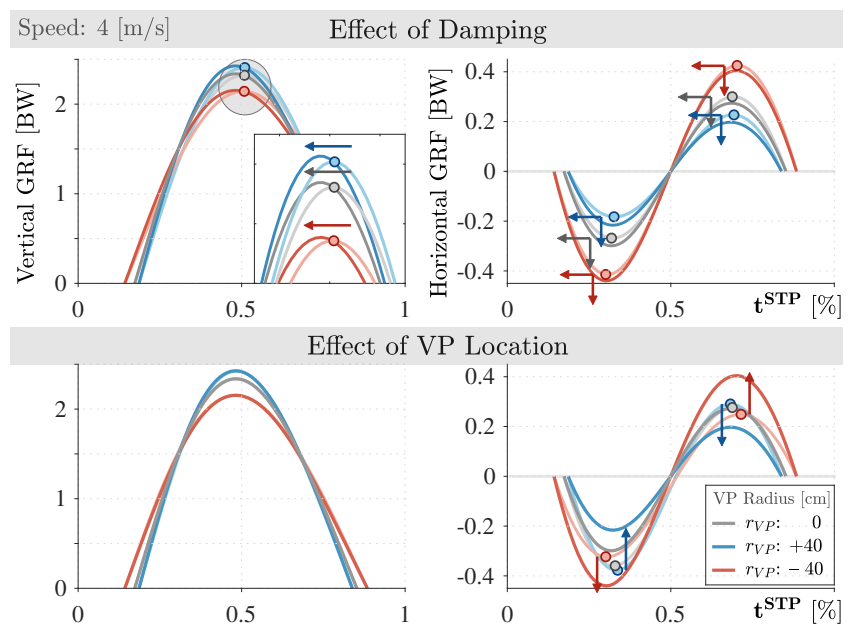

Fig. 5. The light-colored lines represent the GRF, when the component created by damping (top) and hip torque (bottom) are subtracted. We observe a temporal shift in the GRF with the addition of damping. Damping contributes to decelerating over TD-MS and accelerating over MS-TO by changing the $\mathrm{GRF}_{\mathrm{x}}$ magnitude.

\section{Trunk Pitch Oscillations}

Our parameter sweep over $r_{V P}$ reveals that a $\mathrm{VP}_{\mathrm{A}}$ produces backward trunk pitching during the stance phase (Fig. 6 , blue lines). A $\mathrm{VP}_{\mathrm{B}}$ leads to forward trunk motion (red lines), which contradicts with $[13,15]$ that estimates a $\mathrm{VP}_{\mathrm{A}}$ using the GRF measurements of human walking and running. We propose two potential explanations for this discrepancy. First, the trunk pitching motion is measured $180^{\circ}$ out of phase with the whole body pitching in human walking [30]. It hints that the model with a $\mathrm{VP}_{\mathrm{A}}$ might predict the whole body motions instead of the trunk. Second, the GRF data is often cropped at TD-TO due to undesired effects of impact and ankle pushoff [13], which might corrupt the estimation of VP.

The trunk oscillates with a larger amplitude for $\mathrm{VP}_{\mathrm{B}}$ compared to $\mathrm{VP}_{\mathrm{A}}$, given an equal VP radius. The larger amplitude is caused by the larger moment arm of the GRF around the CoM with $\mathrm{VP}_{\mathrm{B}}$. In accordance, at running speeds between 4-10 m/s (Fig. 7), the trunk angular excursion increases up to $5^{\circ}$ for $\mathrm{VP}_{\mathrm{A}}$ and $14^{\circ}$ for $\mathrm{VP}_{\mathrm{B}}$. The mean trunk angular velocity increases up to $38^{\circ} \mathrm{s}^{-1}$ for $\mathrm{VP}_{\mathrm{A}}$ and $-85^{\circ} \mathrm{s}^{-1}$ for $\mathrm{VP}_{\mathrm{B}}$.

The trunk oscillates more at higher speeds, albeit an equal VP target (Fig. 7). As the speed increases (Table II), we see that the leg force ${ }_{F} F_{a}$ increases despite the rise in damping $F_{d p}$, since $F_{s p}$ is significantly larger than $F_{d p}$. The VP method imposes a linear relation between the leg and hip forces. Therefore the GRF gets larger over speed. Higher GRF creates a larger angular moment around the CoM, which

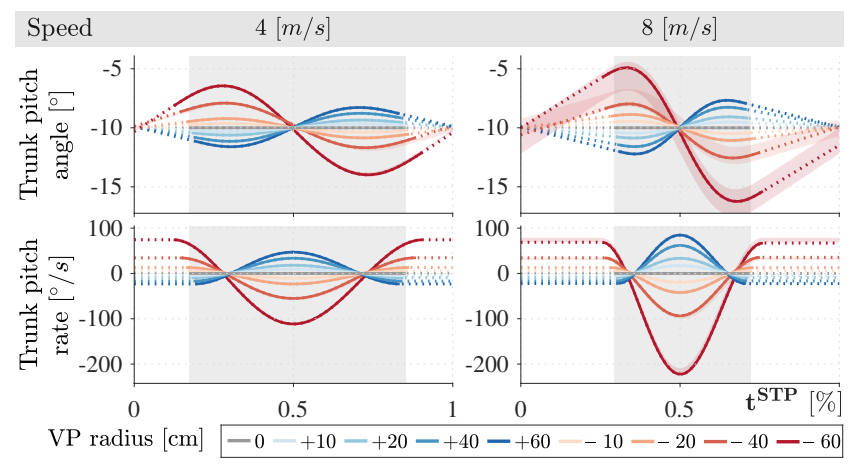

Fig. 6. $\mathrm{VP}_{\mathrm{A}}$ produces backward and $\mathrm{VP}_{\mathrm{B}}$ generates forward trunk pitching The magnitude and rate of pitch oscillations increase with the VP radius.

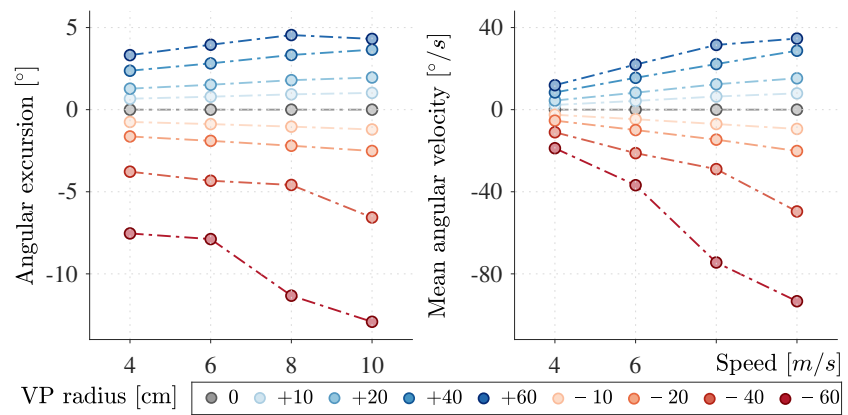

Fig. 7. Trunk angular excursion and peak angular rate increase with speed and increasing absolute VP radius. 
TABLE II. Evolution of the leg forces w.r.t. the increase in forward speed.

\begin{tabular}{c|cccccc}
$\dot{x}\left[\mathrm{~ms}^{-1}\right]$ & $c$ & $\Delta l$ & $i$ & $F_{s p}$ & $F_{d p}$ & ${ }_{F} F_{a}$ \\
\hline 4 & $3 c$ & $\Delta l$ & $i$ & $15 F$ & $F$ & $14 F$ \\
10 & $c$ & $2 \Delta l$ & $6 i$ & $30 F$ & $4 F$ & $26 F$
\end{tabular}

results in a larger trunk oscillation.

\section{Energy Considerations}

In this section, we investigate the energetic consequences our VP target locations. Simulated gaits converge to a periodic steady-state solution, where the system's energy is conserved over steps. However, the energy fluctuates within a single step. The hip torque adds energy to propel to body, and the leg damper depletes this excess energy to prevent the body from accelerating or decelerating. Effectively, the work done by the leg (g) and the hip (h) have the same magnitude and opposite sign (Fig. 99.

In the course of a single step, the hip torque generated by the $\mathrm{VP}_{\mathrm{A}}$ injects energy to the system in the initial stages of the stance phase $(i, \uparrow)$, and removes some energy towards the end (i,, , Fig. 8). The switch occurs after MS (•), and the hip energy end up in a net positive value at the end of the stance phase (๑). For the $\mathrm{VP}_{\mathrm{B}}$ with a radius smaller than $-30 \mathrm{~cm}$, this relation holds as well. When the VP radius gets larger than $30 \mathrm{~cm}\left(\mathrm{VP}_{\mathrm{BL}}\right)$, the VP becomes located below the leg at TD, which changes the sign of the hip torque. Consequently, the hip depletes energy first, reaches to a maximum before MS (•), and injects a greater energy after that $\left(\begin{array}{l}\hat{i} \\ i\end{array}\right)$ to reach to a net positive value $(\bullet)$. The energy $\mathrm{min} /$ maximum gets closer to the MS when $r_{V P}$ increases.

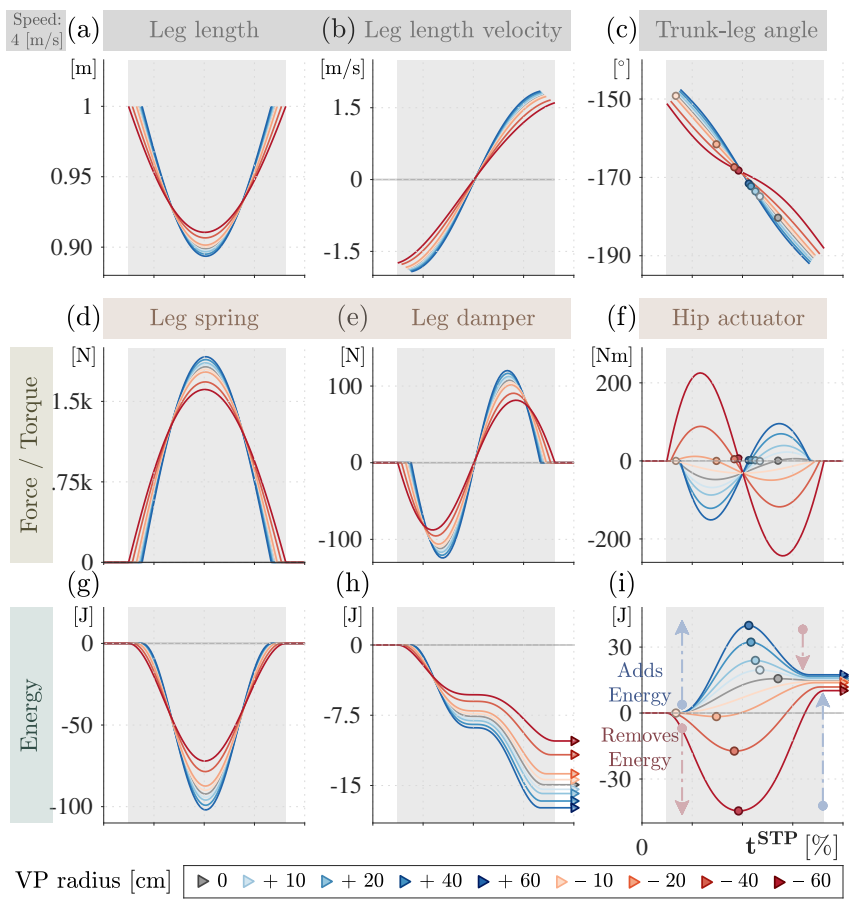

Fig. 8. The hip torque generated by $\mathrm{VP}_{\mathrm{A}}$ injects and removes energy from the system, whereas for $\mathrm{VP}_{\mathrm{BL}}$ the order reverses. Both methods yield a net positive hip work (marked with $(\triangleright, \downarrow)$ ), which leg damper has to remove. The VP radius effects the phase of the energy reversal (marked with $(\bullet, \bullet)$
$\mathrm{VP}_{\mathrm{A}}$ yields higher net hip work $(\mathrm{i}, \bullet)$ than $\mathrm{VP}_{\mathrm{B}}$, which in return requires higher leg damping $(\mathrm{h}, \bullet)$ to sustain the steadystate motion (Fig. 8). This higher damping is provided by a larger $\Delta l$ (a) and $i(\mathrm{~b})$, given that the damping constant $c$ is kept constant for all VP targets at the same speed. The higher $\Delta l$ yields higher spring forces (d) and energies ( $\mathrm{g}$ ) for $\mathrm{VP}_{\mathrm{A}}$.

Our results show that the direction and magnitude of trunk oscillations determine the work distribution between the leg and hip. To analyze these two key factors, (1) we compare $\mathrm{VP}_{\mathrm{A}}$ to $\mathrm{VP}_{\mathrm{B}}$ for the same $\mathrm{VP}$ radius and (2) we vary the $\mathrm{VP}$ radius and analyze its effect for $\mathrm{VP}_{\mathrm{A}}$ and $\mathrm{VP}_{\mathrm{B}}$ separately.

1. Oscillation direction $\left[\mathrm{VP}_{\mathrm{A}}\right.$ vs. $\left.\mathrm{VP}_{\mathrm{B}}\right]$ : $\mathrm{VP}_{\mathrm{A}}$ requires larger leg work $(\mathrm{a}, \mathrm{d}, \mathrm{g})$ and higher net hip work (h, Fig. 9).

2. Oscillation magnitude [var. $r_{V P}$ ]: $\mathrm{VP}_{\mathrm{A}}$ with a larger radius requires higher leg $(\mathrm{a}, \mathrm{d}, \mathrm{g})$ and net hip work $(\mathrm{h})$, whereas its the opposite for $\mathrm{VP}_{\mathrm{B}}$ (Fig. 99). On the other hand, the magnitude of the positive and negative hip work depends on the VP radius and increases with it $(\mathrm{b}, \mathrm{e})$. Consequently, the amount of positive and negative work performed by the hip becomes closer to that of the leg (c,f).

In summary, a VP below the CoM in our running model reduces the leg loading and net hip work (Fig. 9, shaded $\varpi$, $\varpi)$. As the VP is placed further down ( $\square)$, the moment arm of the GRF increases and higher hip torques become necessary to counteract the larger angular moments created. Intuitively, in human running, the GRF forces the trunk to collapse forward at foot contact due to the posterior placement of the hip. If we place the $\mathrm{VP}_{\mathrm{B}}$, the trunk motion conforms to the natural pitching dynamics of the trunk-leg system, which is to rotate forward, and assists the leg to decelerate in fore-aft $(x)$ direction. After MS, the leg dynamics alone would force the trunk to extend when the trunk-leg angle decreases below $180^{\circ}$. $\mathrm{VP}_{\mathrm{B}}$ imposes this transition to happen

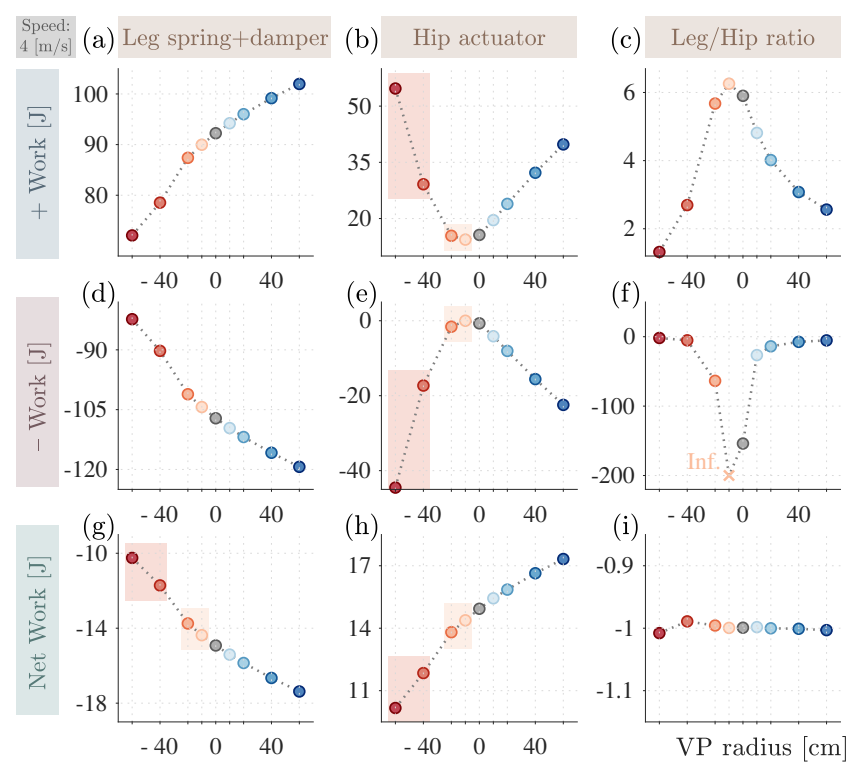

Fig. 9. Figure shows the positive, negative and the net work done by the leg and the hip. $\mathrm{VP}_{\mathrm{B}}$ requires less the leg work and net hip work as opposed to $\mathrm{VP}_{\mathrm{A}}$. However, as $\mathrm{VP}_{\mathrm{B}}$ is placed further down $(\square)$, the absolute positive and negative hip energy requirements increase as well. 
earlier. Meanwhile, it supports the leg in accelerating in $x$. The synergetic work of the hip and leg in $x$ leads to a reduced leg loading. However, as we place the VP further down ( $\square$ ), the increase in the moment arm of the GRF (i.e., increase in angular momentum) dominates and and higher torques are needed to maintain postural stability. Importantly, our results corroborate previous findings in [31], who argue that the trunk posture influences lower extremity biomechanics and energetics by changing the load distribution in lower limbs.

\section{CONCLUSION}

In this work, we investigated how the trunk pitch motion affects the dynamics and energetics of running. We implemented a TSLIP model with a virtual point (VP) either above or below the center of mass (CoM) to generate trunk oscillations. We showed that

(a) VP above the CoM $\left(\mathrm{VP}_{\mathrm{A}}\right)$ produces backward, below the CoM $\left(\mathrm{VP}_{\mathrm{B}}\right)$ produces forward trunk pitch motion,

(b) $\mathrm{VP}_{\mathrm{A}}$ creates smaller trunk angular excursions than $\mathrm{VP}_{\mathrm{B}}$ for the equal VP radius,

(c) the trunk angular excursion and mean trunk angular velocity increase with speed for the same VP radius.

We also analyzed how the VP location modified the work distribution between the hip and leg, and derived two key strategies to leverage the trunk oscillations in favor of energetics. If the control goal is to minimize the leg loading, we suggest a $\mathrm{VP}_{\mathrm{B}}$ with large radius $\left(\mathrm{VP}_{\mathrm{BL}}\right)$, where the hip works synergistically with leg, at the expense of high peak hip torques. If we aim to minimize both the hip and leg work, we suggest $\mathrm{VP}_{\mathrm{B}}$ with a radius smaller than $30 \mathrm{~cm}$. Our results can be extended to different bipedal morphologies with different parameterizations, and have potential implications for humanoid robot control. For instance, one can induce trunk motions and redistribute the joint work to counter motor torque limits or achieve a larger range of motion.

\section{REFERENCES}

[1] M. Raibert, "Hopping on One Leg in the Plane," in Legged Robots That Balance. Cambridge, Massachusetts: The MIT Press, 1986, ch. 2, pp. 29-56.

[2] E. R. Westervelt, J. W. Grizzle, C. Chevallereau, J. H. Choi, and B. Morris, "Systematic Design of Within-Stride Feedback Controllers for Walking," in Feedback Control of Dynamic Bipedal Robot Locomotion. CRC Press, 2007, ch. 6-9.

[3] S. Aminiaghdam, C. Rode, R. Müller, and R. Blickhan, "Increasing trunk flexion transforms human leg function into that of birds despite different leg morphology," Journal of Experimental Biology, vol. 220, no. 3, pp. 478-486, 2017.

[4] L. Heitkamp, "The Role of the Gluteus Maximus on Trunk Stability in Human Endurance Running," Ph.D. dissertation, University of Cincinatti, 2012.

[5] R. N. Hinrichs, "Upper Extremity Function in Running. II: Angular Momentum Considerations," International Journal of Sport Biomechanics, vol. 3, no. 3, pp. 242-263, 1987.

[6] A. G. Schache, K. L. Bennell, P. D. Blanch, and T. V. Wrigley, "The coordinated movement of the lumbo pelvic hip complex during running: a literature review," Gait \& Posture, vol. 10, no. 1, pp. 30-47, 1999.

[7] A. Thorstensson, J. Nilsson, H. Carlson, and M. R. Zomlefer, "Trunk movements in human locomotion," Acta Physiologica Scandinavica, vol. 121, no. 1, pp. 9-22, 1984.

[8] P. de Leva, "Adjustments to Zatsiorsky-Seluyanov's segment inertia parameters," Journal of Biomechanics, vol. 29, no. 9, pp. 1223-1230, 1996.
[9] D. M. Bramble and D. E. Lieberman, "Endurance running and the evolution of Homo," Nature, vol. 432, no. 7015, pp. 345-352, 2004.

[10] H. Kunz and D. A. Kaufmann, "Biomechanical analysis of sprinting: decathletes versus champions," British journal of sports medicine, vol. 15 , no. 3, pp. 177-181, 1981.

[11] K. R. Williams and P. R. Cavanagh, "Relationship between distance running mechanics, running economy, and performance," Journal of Applied Physiology, vol. 63, no. 3, pp. 1236-1245, 1987.

[12] R. Blickhan, "The spring-mass model for running and hopping," Journal of Biomechanics, vol. 22, no. 11, pp. 1217-1227, 1989.

[13] M. H. Maus, "Stabilisierung des Oberkörpers beim Rennen und Gehen," Ph.D. dissertation, Friedrich Schiller University Jena, 1982.

[14] M. A. Sharbafi, "Bioinspired template-based control of legged locomotion," Ph.D. dissertation, Technical University of Darmstadt, 2017.

[15] M. H. Maus, S. W. Lipfert, M. Gross, J. Rummel, and A. Seyfarth, "Upright human gait did not provide a major mechanical challenge for our ancestors," Nature Communications, vol. 1, p. 70, 2010.

[16] J. Vielemeyer, E. Grießbach, and R. Müller, "Ground reaction forces intersect above the center of mass even when walking down visible and camouflaged curbs," The Journal of Experimental Biology, pp. 204-305, 2019.

[17] E. Andrada, C. Rode, Y. Sutedja, J. A. Nyakatura, and R. Blickhan, "Trunk orientation causes asymmetries in leg function in small bird terrestrial locomotion," Proceedings of the Royal Society B: Biological Sciences, vol. 281, no. 1797, 2014.

[18] R. Blickhan, E. Andrada, R. Müller, C. Rode, and N. Ogihara, "Positioning the hip with respect to the COM: Consequences for leg operation," Journal of Theoretical Biology, vol. 382, pp. 187-197, 2015.

[19] P. Scholl, "Modeling Postural Control in Parkinson's Disease," Ph.D. dissertation, Technical University of Darmstadt, 2018. [Online]. Available: http://wiki.ifs-tud.de/abschlussarbeiten/msc/2018\{_\}scholl

[20] S. Shigemi, "ASIMO and Humanoid Robot Research at Honda," in Humanoid Robotics: A Reference, A. Goswami and P. Vadakkepat, Eds. Springer, Dordrecht, 2019, pp. 55-90.

[21] J. I. Yamaguchi, A. Takanishi, and I. Kato, "Development of a biped walking robot compensating for three-axis moment by trunk motion," pp. 561-566, 1993.

[22] A. T. Peekema, "Template-Based Control of the Bipedal Robot ATRIAS," Ph.D. dissertation, Oregon State University, 2015.

[23] S. Rezazadeh and J. W. Hurst, "Toward step-by-step synthesis of stable gaits for underactuated compliant legged robots," in IEEE International Conference on Robotics and Automation (ICRA), 2015, pp. $4532-4538$.

[24] M. A. Sharbafi, C. Maufroy, M. N. Ahmadabadi, M. J. Yazdanpanah, and A. Seyfarth, "Robust hopping based on virtual pendulum posture control," Bioinspiration \& Biomimetics, vol. 8, no. 3, 2013.

[25] T. A. McMahon and G. C. Cheng, "The mechanics of running: How does stiffness couple with speed?" Journal of Biomechanics, vol. 23, pp. $65-78,1990$.

[26] J. Wojtusch and O. von Stryk, "HuMoD - A Versatile and Open Database for the Investigation, Modeling and Simulation of Human Motion Dynamics on Actuation Level," in Proceedings of the IEEERAS International Conference on Humanoid Robots, 2015, pp. 74-79.

[27] I. Abraham, Z. Shen, and J. Seipel, "A Nonlinear Leg Damping Model for the Prediction of Running Forces and Stability," Journal of Computational and Nonlinear Dynamics, vol. 10, no. 5, 2015.

[28] P. J. Bishop, D. F. Graham, L. P. Lamas, J. R. Hutchinson, J. Rubenson, J. A. Hancock, R. S. Wilson, S. A. Hocknull, R. S. Barrett, D. G. Lloyd, and C. J. Clemente, "The influence of speed and size on avian terrestrial locomotor biomechanics: Predicting locomotion in extinct theropod dinosaurs," PLOS ONE, vol. 13, no. 2, 2018.

[29] S. M. Gatesy and A. A. Biewener, "Bipedal locomotion: effects of speed, size and limb posture in birds and humans," Journal of Zoology, vol. 224, no. 1, pp. 127-147, 1991.

[30] R. Müller, C. Rode, S. Aminiaghdam, J. Vielemeyer, and R. Blickhan, "Force direction patterns promote whole body stability even in hipflexed walking, but not upper body stability in human upright walking," Proceedings. Mathematical, physical, and engineering sciences, vol. 473 , no. 2207, 2017.

[31] H.-L. Teng and C. M. Powers, "Influence of Sagittal Plane Trunk Posture on Lower Extermity Biomechanics During Running," Ph.D. dissertation, University of Southern California, 2013. 